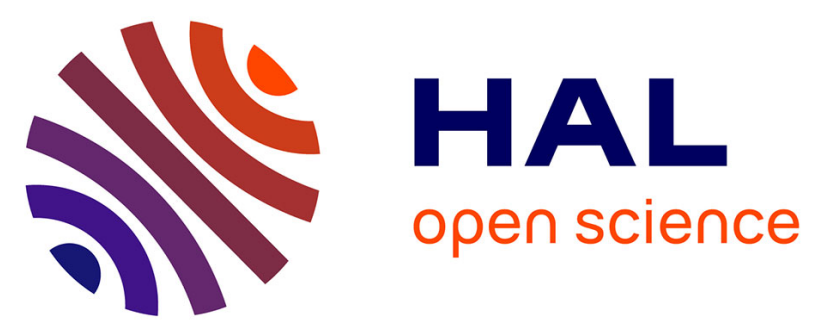

\title{
Fast and Fully Automatic 3D Echocardiographic Segmentation Using B-Spline Explicit Active Surfaces: Feasibility Study and Validation in a Clinical Setting
}

D. Barbosa, T. Dietenbeck, Brecht Heyde, H. Houle, Denis Friboulet, Jan d'Hooge, Olivier Bernard

\section{To cite this version:}

D. Barbosa, T. Dietenbeck, Brecht Heyde, H. Houle, Denis Friboulet, et al.. Fast and Fully Automatic 3D Echocardiographic Segmentation Using B-Spline Explicit Active Surfaces: Feasibility Study and Validation in a Clinical Setting. Ultrasound in Medicine \& Biology, 2013, 39 (1), pp.89-101. 10.1016/j.ultrasmedbio.2012.08.008 . hal-00796930

\section{HAL Id: hal-00796930 \\ https://hal.science/hal-00796930}

Submitted on 20 Nov 2018

HAL is a multi-disciplinary open access archive for the deposit and dissemination of scientific research documents, whether they are published or not. The documents may come from teaching and research institutions in France or abroad, or from public or private research centers.
L'archive ouverte pluridisciplinaire HAL, est destinée au dépôt et à la diffusion de documents scientifiques de niveau recherche, publiés ou non, émanant des établissements d'enseignement et de recherche français ou étrangers, des laboratoires publics ou privés. 


\title{
FAST AND FULLY AUTOMATIC 3-D ECHOCARDIOGRAPHIC SEGMENTATION USING B-SPLINE EXPLICIT ACTIVE SURFACES: FEASIBILITY STUDY AND VALIDATION IN A CLINICAL SETTING
}

\author{
Daniel Barbosa, ${ }^{* \dagger}$ Thomas Dietenbeck, ${ }^{\dagger}$ Brecht Heyde, ${ }^{*}$ Helene Houle, ${ }^{\ddagger}$ Denis Friboulet ${ }^{\dagger}$ \\ JAN D'HOOGE, ${ }^{*}$ and OLIVIER BeRNARD ${ }^{\dagger}$ \\ * Lab on Cardiovascular Imaging and Dynamics, Katholieke Universiteit Leuven, Belgium; ${ }^{\dagger}$ Université de Lyon, CREATIS, \\ CNRS UMR5220, INSERM U630, Université Lyon 1, INSA-Lyon, France; and ${ }^{\ddagger}$ Siemens Ultrasound, \\ Mountain View, CA, USA
}

(Received 28 February 2012; revised 26 June 2012; in final form 13 August 2012)

\begin{abstract}
A novel framework to efficiently deal with three-dimensional (3-D) segmentation of challenging inhomogeneous data in real-time has been recently introduced by the authors. However, the existing framework still relied on manual initialization, which prevented taking full advantage of the computational speed of the method. In the present article, an automatic initialization scheme adapted to 3-D, echocardiographic data is proposed. Moreover, a novel segmentation functional, which explicitly takes the darker appearance of the blood into account, is also introduced. The resulting automatic segmentation framework provides an efficient, fast and accurate solution for quantification of the main left ventricular volumetric indices used in clinical routine. In practice, the observed computation times are in the order of 1 s. (E-mail: daniel.barbosa@uzleuven.be) (C) 2013 World Federation for Ultrasound in Medicine \& Biology.
\end{abstract}

Key Words: Active contours, LV volume analysis, image segmentation, B-splines, 3-D echocardiography.

\section{INTRODUCTION}

Ultrasound imaging faced an important evolution with the introduction of two-dimensional (2-D) matrix transducers in clinical practice. Indeed, three-dimensional (3-D) visualization of the cardiac chambers allows overcoming several important limitations of classical 2-D ultrasound imaging, such as foreshortening, out-ofplane motion and the need of geometric assumptions for volume calculation. Volumetric analysis of left ventricular (LV) function using real-time 3-D echocardiography (RT3DE) has already been proven to be an accurate approach in several comparative studies against the current gold standard (i.e., magnetic resonance imaging [MRI]) (Jenkins et al. 2004; Caiani et al. 2005; Jacobs et al. 2006; Soliman et al. 2007, 2008; Mor-Avi et al. 2008; Muraru et al. 2010). These previous studies have also revealed that the estimation of LV volumes using traditional 2-D imaging has limited accuracy attributable to the limitations introduced by the geometric assump-

Address correspondence to: Daniel Barbosa, Medical Imaging Research Center, Herestraat 49, bus 7003, 3000-Leuven, Belgium. E-mail: daniel.barbosa@uzleuven.be tions required to estimate the volumetric measurements. Furthermore, in these previous studies, interobserver variability was also significantly larger in volumes estimated using 2-D approaches (Jenkins et al. 2004; Caiani et al. 2005; Jacobs et al. 2006). Therefore, the potential of accurate 3-D volumetric assessment of cardiac morphology and function using RT3DE, in addition to the intrinsic benefit of ultrasound imaging (i.e., its cost and portability), makes it a very useful and promising tool for clinical routine.

Nonetheless, and despite the efforts from the research community and medical vendors, 3-D LV segmentation typically remains a time consuming task and heavily relies on user interaction to accurately measure LV volumes. In fact, the amount of time required to accurately extract the most common volumetric indices (end-diastolic volume [EDV], end-systolic volume [ESV], stroke volume [SV], ejection fraction [EF]) from a single examination ranges from $2 \mathrm{~min}$ (Hansegard et al. 2009; Muraru et al. 2010) to $10 \mathrm{~min}$ (Jenkins et al. 2004; Caiani et al. 2005), although approximately 5 min seems to be the most common total analysis time (Jacobs et al. 2006; Soliman et al. 2007; 2008; Mor-Avi et al. 2008). Furthermore, a recent study using 
a state-of-the-art commercial software package shows that although automated LV volume measurements are feasible (nine user clicks at ED and ES for initialization), optimal LV volumetric assessment still requires a significant amount of manual correction (Muraru et al. 2010). This results in doubling the analysis time and also introduces a user-dependent bias. Thus, there is still a significant need for tools allowing automatic fast 3-D LV segmentation, as highlighted in the closing remarks of a recent review focusing on the automated border detection in RT3DE data (Leung and Bosch 2010). This is strengthened by the fact that recent advances in the acquisition techniques are pushing the temporal resolution forward (Madore et al. 2009; Hasegawa and Kanai 2011; Tong et al. 2011), thus enforcing the need for automated methods to handle the increasing amount of data available from a single cardiac cycle.

The authors recently proposed a framework for realtime segmentation of challenging inhomogeneous 3-D data (Barbosa et al. 2012a). This method uses B-spline explicit active surfaces (BEAS) to recover objects from volumetric data, allowing the use of global or local region-based segmentation energies to evolve the contour. A pipeline designed to segment RT3DE data using BEAS was recently developed. In spite of obtaining promising results, the existing semiautomated algorithm relies on manual input (six clicks per 3-D volume) to fit an ellipsoid used to initialize the segmentation algorithm (Barbosa et al. 2011, 2012b). This fact intrinsically introduces userdependent bias. Moreover, manual initialization accounts for the vast majority $(\sim 95 \%)$ of the total analysis time. Thus, a fast and automatic initialization method would be of great clinical value. This would overcome any variability introduced by the manual initialization, while taking full advantage of the real-time ability of BEAS.

Several attempts have recently been made to tackle the initialization dependence of energy-based image segmentation strategies. Li and Acton have proposed an initialization framework aiming to recover the underlying external energy field from the external (image) force field, using then isolines of minimum energy to initialize the segmentation process ( $\mathrm{Li}$ and Acton 2008). Tauber et al. have proposed the concept of centers of divergence to solve the initialization dependence of active contours (Tauber et al. 2010). Regarding cardiac segmentation problems, van Stralen et al. have proposed a method for automatic estimation of both LV orientation and mitral valve detection (van Stralen et al. 2008).

The originality of the proposed method is twofold: first, a new segmentation energy that takes explicitly into account the darker appearance of the blood pool is introduced, contributing to a more robust and accurate segmentation. Second, getting inspired by the work of van Stralen et al. (2008), a new algorithm based on a robust phase-based edge detector and a fast ellipsoid fitting procedure is proposed. This allows keeping the computational burden of the initialization method around $1 \mathrm{~s}$ in a $\mathrm{C}++$ implementation. The fully automatic segmentation method as such obtained is then validated using clinical volumetric ultrasound data.

\section{METHODS}

\section{3-D LV real-time segmentation using soft prior information}

The segmentation framework (BEAS) used in the present work has been recently proposed by the authors to allow real-time segmentation of challenging inhomogeneous 3-D data (Barbosa et al. 2012a). The fundamental concept of this method is to regard the boundary of an object as an explicit function, where one of the coordinates of the points within the surface is given explicitly from the remaining coordinates. Such explicit relation can be mathematically defined as:

$$
\psi: \mathbb{R}^{n-1} \mapsto \mathbb{R}, \quad\left(x_{2}, \ldots, x_{n}\right) \mapsto x_{1}=\psi\left(\mathbf{x}^{*}\right),
$$

where $\mathbf{x} \in \mathbb{R}^{n}$ is a point of coordinates $\left\{x_{1}, \ldots, x_{n}\right\}$ in an $n$-dimensional space and $\mathbf{x}^{*} \in \mathbb{R}^{n-1}$ is a point of coordinates $\left\{x_{2}, \cdots, x_{n}\right\}$ in the associated $(n-1)$-dimensional subspace.

In this framework, the explicit function $\Psi$ is expressed as the linear combination of B-spline basis functions (Unser 1999):

$$
x_{1}=\psi\left(x_{2}, \cdots, x_{n}\right)=\sum_{\mathbf{k} \in \mathbf{z}^{n-1}} c[\mathbf{k}] \beta^{d}\left(\frac{\mathbf{x}^{*}}{h}-\mathbf{k}\right),
$$

where $\beta^{d}(\cdot)$ is the uniform symmetric $n-1$-dimensional B-spline of degree $d$. This function is separable and is built as the product of $n-1$ one-dimensional B-splines, so that $\beta^{d}\left(\mathbf{x}^{*}\right)=\prod_{j=2}^{n} \beta^{d}\left(x_{j}\right)$. The knots of the B-splines are located on a rectangular grid defined on the chosen coordinate system, with a regular spacing given by $h$. The coefficients of the B-spline representation are gathered in $c[\mathbf{k}]$. Note that this representation follows what has been initially proposed for the level-set framework by Bernard et al. (2009).

The surface will evolve toward the object boundaries by minimization of a segmentation energy functional. Typically, the localized Yezzi energy, as proposed in Lankton and Tannenbaum (2008), has been used:

$$
E_{L}=\int_{\Omega} \delta_{\phi}(\mathbf{x}) \int_{\Omega} B(\mathbf{x}, \mathbf{y}) F_{L}\left(\mathbf{y}, H_{\phi}(\mathbf{y})\right) \mathrm{d} \mathbf{y} \mathrm{d} \mathbf{x}
$$

and

$$
F_{L}\left(\mathbf{y}, H_{\phi}(\mathbf{y})\right)=-\left(u_{x}-v_{x}\right)^{2}
$$


where $F_{L}\left(\mathbf{y}, H_{\phi}(\mathbf{y})\right)$ is an image criterium and $B(\mathbf{x}, \mathbf{y})$ corresponds to a mask function in which the local parameters that drive the evolution of the interface are estimated. $\delta_{\phi}(\mathbf{x})$ and $H_{\phi}(\mathbf{x})$ are the Dirac and Heaviside operators respectively applied to the level set function $\phi(\mathbf{x})=\psi\left(\mathbf{x}^{*}\right)-x_{1}$, which is defined over the image domain $\Omega$. Note that $u_{x}$ and $v_{x}$ are the local mean intensities inside and outside the interface $\Gamma$, computed along the normal direction as suggested in Barbosa et al. (2012a).

Since the blood pool is usually darker than the myocardial tissue, we proposed to introduce a small change in the original function by:

$$
F_{L}\left(\mathbf{y}, H_{\phi}(\mathbf{y})\right)=\left(u_{x}-v_{x}\right) .
$$

Note that whenever $u_{x}>v_{x}$, this segmentation energy will have a positive value, which is penalized in the proposed minimization strategy. It is important to stress that the proposed energy is intrinsically local, and thus will converge to a local minimum. This is in line with the goal of the proposed method, which consists in obtaining the optimal boundary between the blood pool and the myocardial tissue, disregarding the remaining contents of the image. Therefore, the minimization of the proposed energy will converge to a local optimum, corresponding to the interface maximizing the signed separation between the local means around the interface (i.e., enforcing the mean outside to be greater than the one inside). It should also be noted that both $u_{x}$ and $v_{x}$ are bounded by the image values range, which imposes a lower bound on eqn (3).

The proposed energy can be directly minimized wrt. the B-spline coefficients, as shown in the Appendix, using the following evolution equations:

$$
\begin{gathered}
c[\mathbf{k}]^{(t+1)}=c[\mathbf{k}]^{(t)}-\lambda \frac{\partial E_{L}}{\partial c[\mathbf{k}]^{(t)}}, \\
\frac{\partial E_{L}}{\partial c\left[\mathbf{k}_{\mathbf{i}}\right]}=\int_{\Gamma}\left(\frac{\left(\bar{I}\left(\mathbf{x}^{*}\right)-u_{x}\right)}{A_{u}}+\frac{\left(\bar{I}\left(\mathbf{x}^{*}\right)-v_{x}\right)}{A_{v}}\right) \beta^{d}\left(\frac{\mathbf{x}^{*}}{h}-\mathbf{k}_{i}\right) \mathrm{d} \mathbf{x}^{*},
\end{gathered}
$$

where $A_{u}$ and $A_{v}$ are the areas inside and outside the interface used to estimate the local means $u_{x}$ and $v_{x}$, respectively. For clarity sake, $\bar{I}\left(\mathbf{x}^{*}\right)$ corresponds to the image value at the position $\mathbf{x}=\left\{\psi\left(\mathbf{x}^{*}\right), x_{2}, \cdots, x_{n}\right\}$. Further details on the BEAS framework can be found in Barbosa et al. (2012a).

In the present work, the $\Psi$ function was defined in spherical coordinates and the number of points used to discretize the interface, denoted as $N_{b}$, were $24 \times 16$ along the zenithal (i.e., longitudinal) and azimuthal (i.e., circumferential) directions, respectively. The scale parameter $h$ was fixed to 2 , while the size of the neighbor- hood $B(x, y), \rho$, was set to 16 . In the Results section, the influence of the choice of these parameters is addressed and the influence of the variation of these parameters is investigated.

\section{Automatic initialization algorithm}

The key steps of the proposed algorithm are the sequential detection of the left ventricular long axis (LAX) and mitral valve plane. Once this information is available, we take advantage of the information gathered in these two first steps to fit an ellipsoid to the detected endocardial boundaries. The overview of the key processing blocks of the algorithm are shown in Figures 1 and 2.

\section{Image preprocessing}

The first step for the automatic initialization of a 3-D ultrasound volume is to detect the myocardial boundaries using edge operators in several C-planes (i.e., planes parallel to the transducer surface), perpendicular to the acquisition axis. Given that ultrasound images possess low contrast between the blood pool and the myocardial tissue, it is proposed to include a robust, phase-based edge detector, because of its intrinsic invariance to intensity. The local phase extracted using monogenic signal, recently applied to 3-D ultrasonic data by Rajpoot et al. (2009), has a remarkable performance in the detection of the myocardial boundaries in low quality ultrasound data, even in low contrast areas. This edge detector is applied to each of the 2-D C-planes taken $5 \mathrm{~mm}$ apart.

Since the endocardium is approximately a circle in these 2-D C-planes, low level computer vision methods can be employed to robustly find the most likely position for the center of the LV cavity. To this end, the Hough transform for circles (HTc) is employed. This transform will return the probability of a given position $(x, y)$ in the 2-D C-plane image being the center of a circle of radius $r$. By concatenating the output of the HTc, a 4D $(x, y, z, r)$ circle center probability matrix, $P_{c}$, can be generated. To keep $r$ in a physiologically relevant range, $\left[r_{\min }, r_{\max }\right]$ was set to $[15,35] \mathrm{mm}$. A schematic diagram of the steps taken in the image preprocessing block are shown in Figure 1a. The multiscale phase-based edge detector algorithm used wavelengths of 24, 30 and 36 pixels, as suggested in the original submission by Rajpoot et al. (2009). Its result was normalized to its maximum value and thresholded using $T h=0.05$ to obtain a binary edge map detector prior to circle detection.

\section{Long axis detection}

To reduce the computational complexity of the long axis detection step, $P_{c}$ was transformed into a 3-D matrix, $P_{\max }$, by taking into account only the maximum probability value along the $r$ direction. Subsequently, multidimensional dynamic programming (MDP) was used to 
a

b C

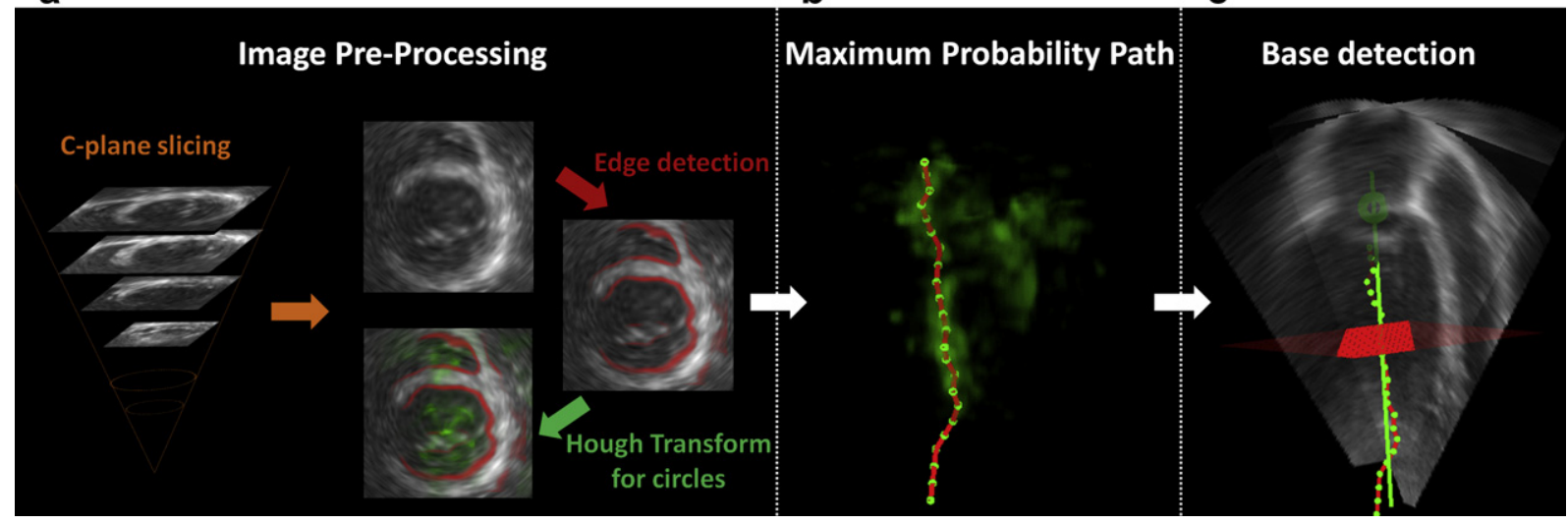

Fig. 1. Left ventricular long axis (LAX) and base detection: (a) Image pre-processing (red - edge indicator, green - circle center probability); (b) Maximum probability path, displayed over $P_{\max }$; (c) Base detection (red - sliding averaging plane; green ball detected base position).

find the optimal path that maximizes the center probabilities along the $Z$ direction (Uzumcu et al. (2006)). For each C-plane slice, $P_{\max }$ can be seen as a density map and thus, thanks to the MDP algorithm, a path corresponding to a set of positions on each of the 2-D C-planes that have high probability of being a center of a circle will be found. Note that during the MDP estimation of the optimal path, connectivity constraints are imposed to penalize the variation of $\mathrm{XY}$ coordinates, to have a smoother path. Through the positions of the path found with MDP, a straight line is fit to determine the left ventricular long axis. A demonstrative example of $P_{\max }$ (in green transparency) and the optimal path found with MPD (green dots connected with red segments) are shown in Figure $1 b$.

\section{Base detection}

After LAX detection, a perpendicular square plane of size $r_{\max }$ was slid along the LAX. For each position, an average gray level was taken by averaging intensities over this square plane. Given that the base plane corresponds both to an area of brighter appearance and to a dark-to-bright transition, a simple merit function, whose maximum corresponds to the base position, was built as:

$$
g(z)=I_{L A X}(z)+\left(I_{L A X}(z)-I_{L A X}(z-\zeta)\right),
$$

where $I_{L A X}(z)$ corresponds to the average gray level of the square plane perpendicular to the LAX at the depth $z$ and $\zeta$ is a distance parameter used to assess the expected darkto-bright transition. $\zeta$ was set to $0.5 \mathrm{~cm}$ in all the experiments. Note that the first term in eqn (8) accounts for the brighter appearance of the valve plane and the second term accounts for the variation in the gray level intensity attributable to the blood-valve plane transition.

\section{Ellipsoid fitting}

$P_{c}$ was sampled along the estimated LAX resulting in a 2-D feature map, $F M(z, r)$, which will correspond to the probability of the estimated LAX positions being the center of a circle of radius $r$. Next, this feature map

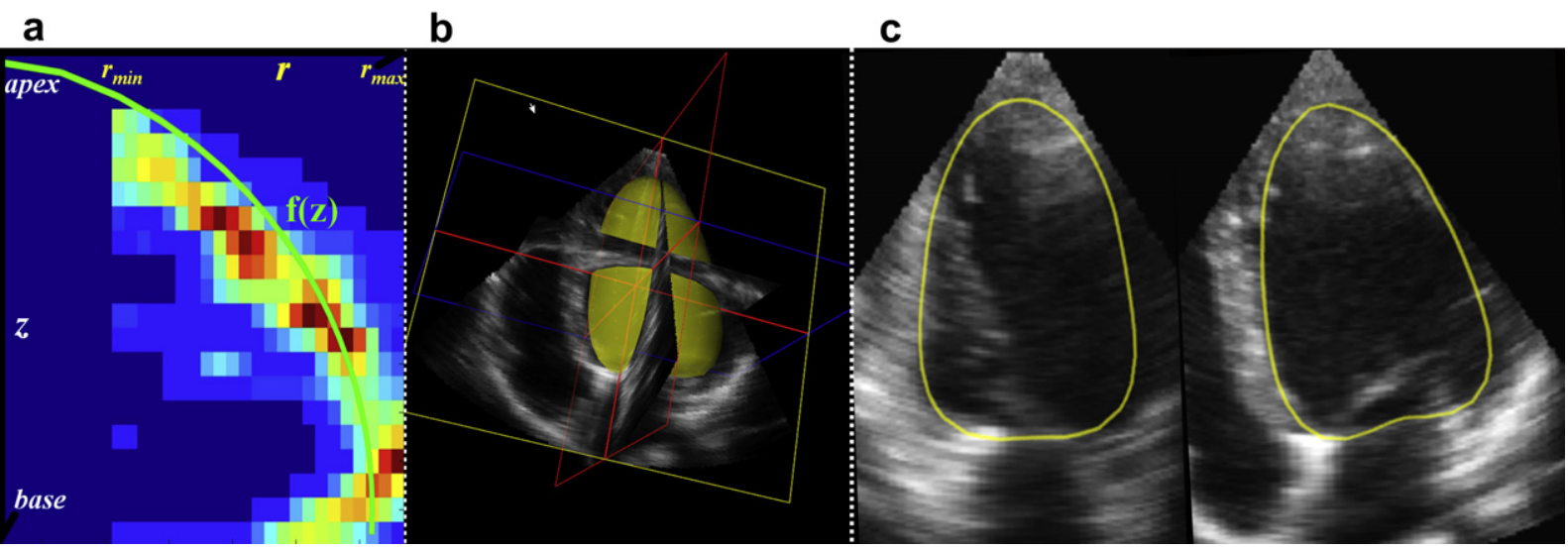

Fig. 2. Ellipsoid fitting: (a) Feature map and optimal ellipsoid fit in cylindrical coordinates (green); (b and c) Initial surface for the 3-D image segmentation step. 
needs to be converted to a true 3-D model that can be used to initialize the segmentation algorithm. To this end, we propose to formulate the ellipsoid fitting problem using its parametric equation defined in a cylindrical coordinate system. It should also be noted that all the required data was already computed in the long axis detection step, being thus a very computationally efficient solution. Consider the ellipsoid parametric equation:

$$
\frac{x^{2}}{a^{2}}+\frac{y^{2}}{b^{2}}+\frac{z^{2}}{c^{2}}=1 .
$$

Assuming that $a=b$ (i.e., that the ellipsoid is actually a spheroid), it can be easily shown that $x^{2}+y^{2}$ is actually the radius of the circle that results from the intersection of the ellipsoid and a plane perpendicular to the $Z$ axis. Thus, the radius of each $\mathrm{z}$-cut of the ellipsoid can be expressed as:

$$
r=f(z)=a \sqrt{1-\frac{z^{2}}{c^{2}}} .
$$

The apex was fixed at $2 \mathrm{~cm}$ from the probe to provide a rough estimate of the longitudinal measure for the LVellipsoid fitting process. From all the plausible curves $\mathrm{f}(\mathrm{z})$ $\left(a \in\left[r_{\text {min }}, r_{\text {max }}\right]\right.$ and $c \leq d_{L A X}$, where $d_{L A X}=$ base $\left._{Z}-2 c m\right)$, the one maximizing the sum of the radius probability along the LAX was selected, as shown in Figure 2a. Once an automatically fitted ellipsoid is available for the end-diastolic (ED) frame, it will be used as initialization of the segmentation algorithm.

\section{End-systolic initialization}

Since in clinical practice physicians are interested in both end-diastolic and end-systolic volumes (EDV, ESV) and functional parameters extracted from these, a second initialization is needed for the end-systolic (ES) frame. To provide a fast initialization and to keep coherence with the segmentation result at end-diastole, a uniform deformation is applied to the segmented LV at ED to initialize the ES frame, as illustrated in Figure 3.

The segmentation result at ED, namely the mesh $\mathbf{M}$ resulting from the sampling of $\psi(\theta, \phi)$, is used as the input of the aforementioned deformation scheme:

$$
\begin{aligned}
& \mathbf{M}=(x=\psi(\theta, \phi) \cos (\theta) \sin (\phi), \\
& y=\psi(\theta, \phi) \sin (\theta) \sin (\phi), z=\psi(\theta, \phi) \cos (\phi)),
\end{aligned}
$$

where $\psi(\theta, \phi)$ is the explicit surface function, which has been discretized in a rectangular grid over the azimuthal and zenithal angles, $\theta$ and $\phi$ respectively. Given that the reference $\mathrm{Z}$-axis of the coordinate system was aligned with the detected LV long axis, $\mathbf{M}$ can be compressed circumferentially simply by:

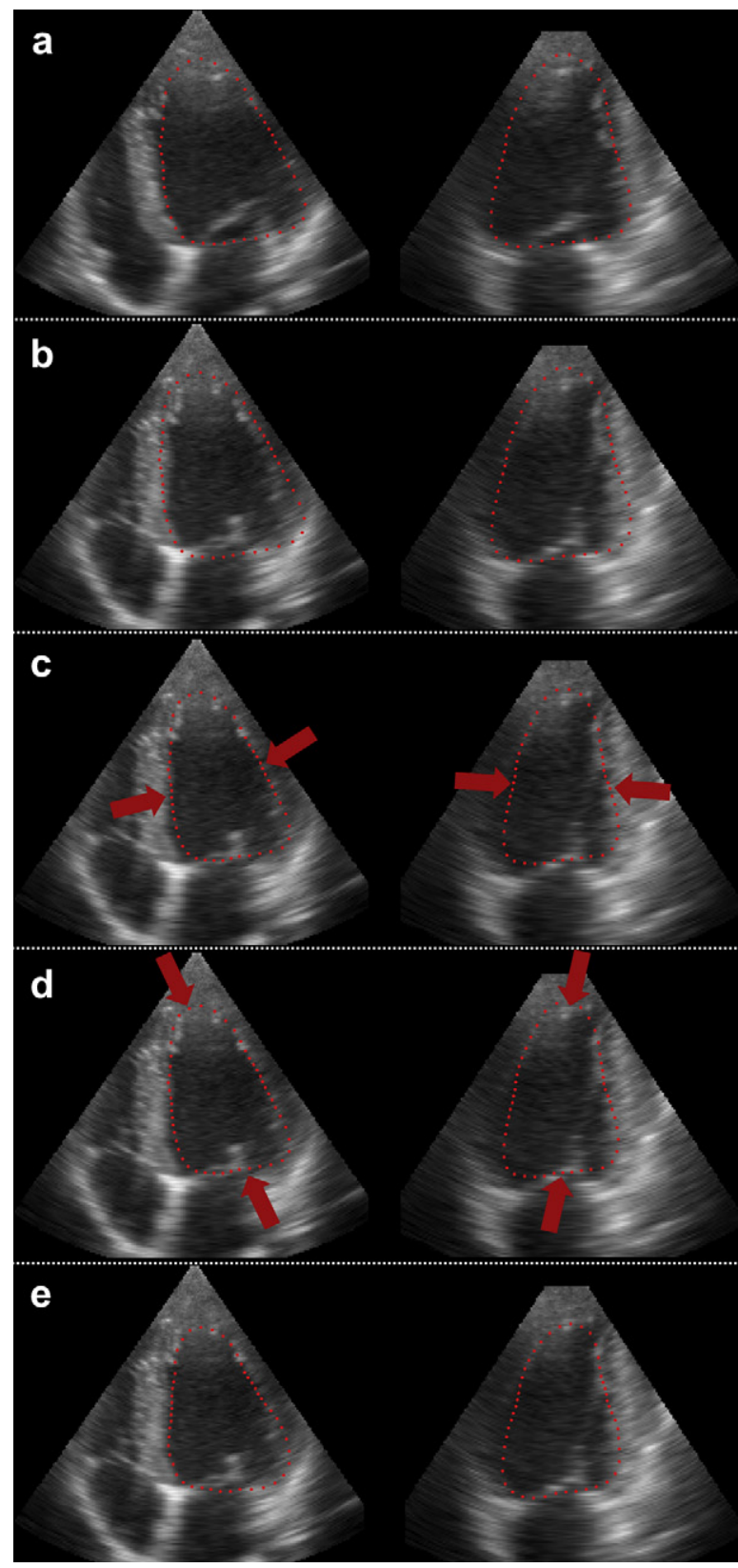

Fig. 3. End-systolic initialization scheme: The contours arising from the automatic segmentation of the end-diastolic frame (a) are overlaid in the ES 3-D volume (b), being then deformed circumferentially (c) and longitudinally (d) toward a state that maximizes the local contrast around the interface. Then, Bspline explicit active surfaces (BEAS) is applied to determine the final segmentation result (e).

$$
\begin{aligned}
\mathbf{M}_{\mathbf{c}}= & \left(\left(1-\varepsilon_{c}\right) \psi(\theta, \phi) \cos (\theta) \sin (\phi)\right. \\
& \left.\left(1-\varepsilon_{c}\right) \psi(\theta, \phi) \sin (\theta) \sin (\phi), \psi(\theta, \phi) \cos (\phi)\right)
\end{aligned}
$$

The range of $\varepsilon_{c}$ has been set to $[10,30] \%$, in agreement with the circumferential deformation ranges previously reported for the left ventricle (Bogaert and Rademakers 2001; Saito et al. 2009; Barbosa et al. 2010). Using the aforementioned segmentation energy 
Table 1. Clinical and echocardiographic characteristics of the study population ( $\mathrm{N}=24, \mu \pm \sigma$, [Range])

\begin{tabular}{|c|c|}
\hline Population details & \\
\hline Age (years) & $46.4 \pm 22.1[11-83]$ \\
\hline HR (bpm) & $67.0 \pm 19.5[54-111]$ \\
\hline \multicolumn{2}{|l|}{ LV parameters } \\
\hline EDV (mL) & $137.3 \pm 49.7$ [50.1-239.7] \\
\hline $\operatorname{ESV}(\mathrm{mL})$ & $66.5 \pm 35.7[25.8-157.3]$ \\
\hline $\mathrm{EF}(\%)$ & $52.7 \pm 11.8[21.8-67.8]$ \\
\hline \multicolumn{2}{|l|}{ Clinical diagnosis information } \\
\hline Healthy $(\%)$ & 20 \\
\hline Valvular disease $(\%)$ & 52 \\
\hline Congenital heart defect $(\%)$ & 8 \\
\hline Apical aneurysm $(\%)$ & 4 \\
\hline Ischemical cardiomyopathy $(\%)$ & 4 \\
\hline Hypertrophic cardiomyopathy (\%) & 4 \\
\hline Atrial fibrillation $(\%)$ & 4 \\
\hline Other $(\%)$ & 12 \\
\hline
\end{tabular}

$\mathrm{EDV}=$ end-diastolic volume; $\mathrm{ESV}=$ end-systolic volume; $\mathrm{EF}=$ ejection fraction; $L V=$ left ventricular.

functional eqn (3), we can assess the energy associated to the circumferential deformation applied to $\mathbf{M}$ as a function of $\varepsilon_{c}$. Once the entire deformation range was assessed (in $1 \%$ variation steps), the optimal circumferential, $\varepsilon_{c_{o p t}}$ deformation can be taken as the one minimizing the proposed energy functional.

Once the model has been deformed circumferentially, it is subsequently deformed in the longitudinal direction in an analogous manner, setting the range of $\varepsilon_{l}$ to $[10,30] \%$ (Bogaert and Rademakers 2001; Saito et al. 2009; Kuznetsova et al. 2008; Barbosa et al. 2010). However, if $\varepsilon_{c_{\text {opt }}}<15 \%$, the $\varepsilon_{l}$ range was constrained to $[5,15] \%$, since such low circumferential deformation reveals that global LV dysfunction may exist.

Using the proposed update scheme, it can be guaranteed that the initialization at ES corresponds to a low segmentation energy state, being thus most likely in the convergence basin of the desired energy optimum.

\section{Experiments}

Data acquisition and reference values. The proposed framework was tested using real-time 3-D echocardiography exams acquired with a Siemens Acuson SC2000 (Siemens Ultrasound, Mountain View, CA, USA), using a 4Z1c matrix transducer. Volume sequences were acquired from an apical window and the sonographer aimed at the inclusion of the entire LV within the pyramidal field of view. All the data used in the present

Table 2. Image quality evaluation

\begin{tabular}{llcc}
\hline Image quality & Expert 1 & Expert 2 & Expert 3 \\
\hline Poor (\%) & 20.83 & 25.00 & 29.17 \\
Fair (\%) & 37.5 & 50.00 & 29.17 \\
Good (\%) & 41.67 & 25.00 & 41.67 \\
\hline
\end{tabular}

work was acquired with the informed consent of all the subjects involved and was anonymized prior to analysis. This study was performed according to the ethical principles for medical research involving human subjects of the World Medical Association's declaration of Helsinki. Each sequence was manually segmented by three clinical experts, using a semiautomated segmentation tool (eSie LVA pre-release software; Siemens Ultrasound) with subsequent manual refinement of the segmentation results, corresponding therefore to a computer-aided manual segmentation of the RT3DE frames. The reference LV volumetric parameters (end-diastolic volume [EDV], end-systolic volume [ESV], stroke volume [SV] and ejection fraction $[\mathrm{EF}]$ ) were estimated as the average of the values estimated by the three experts.

Data processing and analysis. Image quality was rated by three experts as poor, fair or good in accordance to the percentage of the myocardial wall clearly visible in the image $(<60 \%, 60 \%-75 \%,>75 \%)$, the contrast between the blood pool and the tissue and the presence of severe image artifacts.

At first, a semiautomated version of the proposed method was used, where two users were asked to provide six clicks in the ED and ES frames, used to fit an ellipsoid in each frame. Note that these users are not the same experts that provided the reference values, being, thus, blinded to the reference manual segmentation process. By providing the same initialization to the segmentation algorithm, it was possible to clearly highlight the effect of taking the darker appearance of the blood explicitly into account in the segmentation energy in contrast to the regular, symmetric local Yezzi energy, where the sign of the local contrast is not taken into account.

Subsequently, the automatic initialization algorithm previously described was coupled with the BEAS framework, using the proposed segmentation energy, providing therefore an integrated framework for fully automatic 3-D segmentation of the left ventricle.

For accuracy and agreement assessment, linear regression and Bland-Altman analysis (Bland and Altman 1986) were performed between the BEAS values (both for manual and automatic initialization) and the reference ones (average of the volumetric indices extracted manually by the three experts).

Parameter sensitivity analysis. The William's test was used to quantify the influence of the parameter variation. This statistical test allows assessing whether the results from our algorithm are within the agreement limits of the experts' manual references. When the William's index (WI) is greater or equal to one, it indicates that the LV indices estimated with the automated method differ from the manual LV indices as much as the LV indices differ from one manual expert to another. 

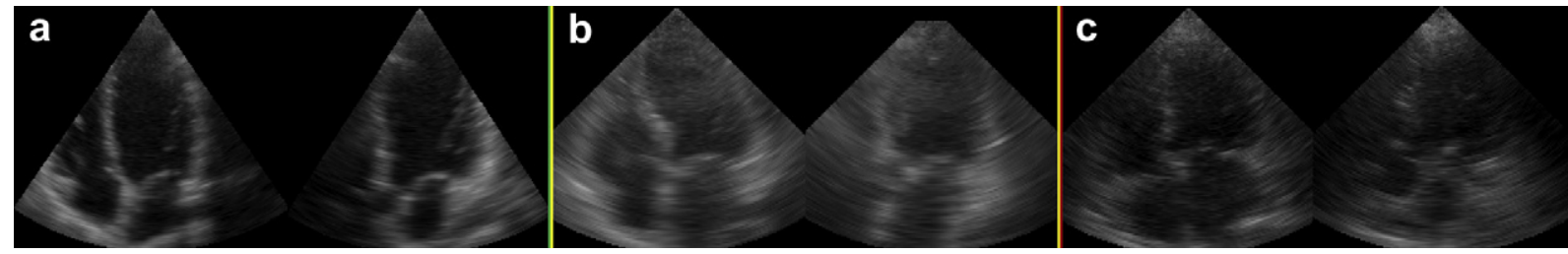

Fig. 4. Variation of image quality of the analyzed dataset (expert consensual classification to: $a=$ good; $b=$ fair; $c=$ poor).

In fact, whenever the upper bound of the $95 \%$ confidence interval of WI $\left(W I_{u p 95 \%}\right)$ is greater than one, there is no statistical evidence that the agreement between the automated method and the reference observers is less than the reference interobserver agreement. Further details regarding this test can be found in the original work of Chalana and Kim (1997).

Since the initialization algorithm is strongly dependent on the initial edge detection step, the effect of the variation of the wavelengths of the filters used in the phase-based edge detection was studied, as well as the threshold used to generate the binary map used subsequently on the Hough transform for circles. The parameters studied for the segmentation algorithm were the size of the grid used to discretize the spherical domain where $\Psi$ is defined, as well as the size of the neighborhood $\rho$ used to estimate the local means.

To define an appropriate range of variation where the segmentation performance is not impaired, $W I_{u p 95 \%}$ was used. The segmentation was considered successful for a given parameter setting if $W I_{u p 95 \%}>1$ for EDV, ESV, $\mathrm{SV}$ and EF simultaneously. To define the initial ranges where the parameter variation influence would be evaluated, the aforementioned optimal parameters were used and the initial range was defined by a perturbation of $50 \%$ of the numeric value of the parameter. In case $W I_{u p 95 \%}>1$ for this interval, the range was increased until the condition for accurate segmentation performance was not met.

\section{RESULTS}

Patient demographics and image quality analysis

Twenty-four examinations were used in the present study, taken randomly from an existing database whereas the only inclusion criteria was to have the LV included in the image pyramid. The dataset comprised both healthy individuals and patients with different pathologies, including valvular disease, congenital heart defects and ischemic/dilated cardiomyopathy with severely distorted LV geometry. An overview regarding the clinical details of the dataset used in the present study is given in Table 1.

The details regarding the expert assessment of the image quality are given in Table 2. Moreover, typical volumes with varying image quality and that gathered consensus on the experts' image quality classification are presented in Figure 4.

\section{Proposed energy term}

The overview of the results for the comparison between the LV volumetric indices extracted using the semiautomated (manual initialization) approach can be found in Table 3. The proposed segmentation energy shows a stronger correlation with the reference values than the original local Yezzi functional for all the cardiac volumetric indices. Bland-Altman analysis shows tighter limits of agreement for proposed segmentation energy, although a higher bias in EDV was observed when compared with the original Yezzi functional. Figure 5 shows a comparison of the contour evolution with the original local Yezzi functional and the proposed segmentation energy, for the same initialization.

\section{Automatic initialization algorithm}

Table 4 provides an overview of the LV volumetric indices extracted using the fully automatic approach. The proposed framework for automatic LV volumetric assessment shows a strong correlation with the reference values $(\mathrm{R}=0.971,0.972,0.938$ and 0.907 for EDV, ESV, $\mathrm{SV}$ and EF, respectively). Bland-Altman analysis revealed small bias and none of these was statistically

Table 3. Comparison of the segmentation performance using manual initialization and different segmentation energies

\begin{tabular}{|c|c|c|c|c|c|c|c|c|}
\hline \multirow{2}{*}{$\begin{array}{l}\text { Initialization/ } \\
\text { segmentation }\end{array}$} & \multicolumn{4}{|c|}{ Semiautomated analysis 1 (manual/local Yezzi ${ }^{\text {Lankton } 2008 \text { ) }}$} & \multicolumn{4}{|c|}{ Semiautomated analysis 2 (manual/proposed energy) } \\
\hline & $\mathrm{EDV}(\mathrm{mL})$ & $\mathrm{ESV}(\mathrm{mL})$ & $\mathrm{SV}(\mathrm{mL})$ & $\mathrm{EF}(\%)$ & $\mathrm{EDV}(\mathrm{mL})$ & $\mathrm{ESV}(\mathrm{mL})$ & $\mathrm{SV}(\mathrm{mL})$ & $\mathrm{EF}(\%)$ \\
\hline Correlation coefficient $(\mathrm{R})$ & 0.934 & 0.887 & 0.720 & 0.800 & 0.954 & 0.951 & 0.932 & 0.843 \\
\hline $\operatorname{Bias}(\mu)$ & -3.91 & $-18.4^{*}$ & $-14.5^{*}$ & $11.63^{*}$ & $7.35^{*}$ & 0.77 & $6.58^{*}$ & $3.12 *$ \\
\hline $\operatorname{LOA}(\mu \pm 1.96 \sigma)$ & {$[-39.1,31.3]$} & {$[-63.9,27.1]$} & {$[-35.0,64.0]$} & {$[-13.7,36.9]$} & {$[-22.4,37.5]$} & {$[-21.3,22.9]$} & {$[-13.6,26.7]$} & {$[-9.72,16.0]$} \\
\hline
\end{tabular}

$\mathrm{EDV}=$ end-diastolic volume; $\mathrm{ESV}=$ end-systolic volume; $\mathrm{SV}=$ stroke volume; $\mathrm{EF}=$ ejection fraction; $\mathrm{LOA}=\mathrm{Bland}$-Altman's limits of agreement. $* p<0.05$, paired $t$-test. 


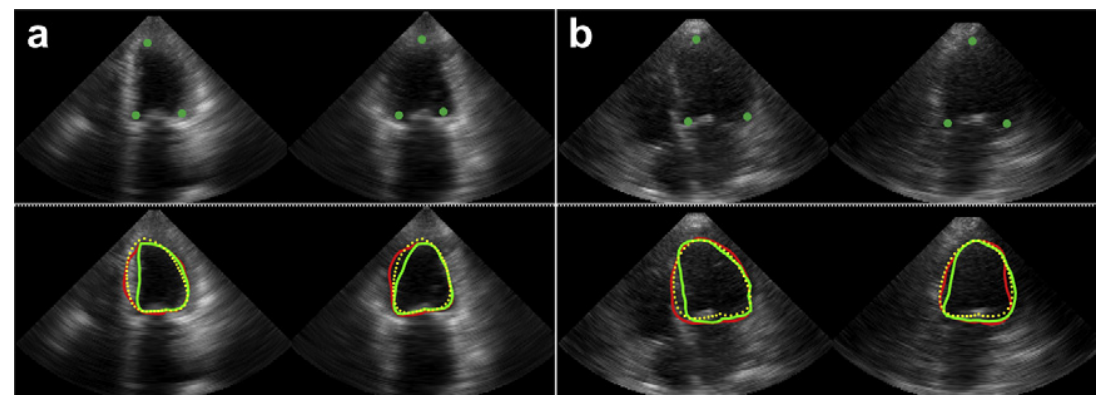

Fig. 5. Comparison between the interface evolution with proposed segmentation energy (green) and the classical localbased Yezzi energy term (red), upon the same initialization (yellow) by the user (three clicks in two planes, top row).

significant for the fully automatic approach, whereas the bias for the EDV, SV and EF were statistically significant (paired $t$-test, $p<0.05$ ) for the semiautomated framework using the proposed energy (Table 3). The BlandAltman plots for all the assessed cardiac volumetric indices with the proposed automatic approach are shown in Figure 6. Figure 7 shows three datasets with varying image quality and its respective automatic initializations compared with the ones provided manually by two observers.

\section{Parameter sensitivity analysis}

Figure 8 illustrates the influence of the variation of each parameter in the fully automatic segmentation performance. The upper bound of the $95 \%$ confidence interval of the William's index $\left(W I_{u p 95 \%}\right)$ was greater than 1 simultaneously for all the computed LV volumetric indices when the initialization algorithm ran with a edgethreshold value below 0.07 and also while the wavelengths of the filters used in the multiscale phase-based detector block were in the interval [(12 15 18); (28 35 42)]. Moreover, $W I_{u p 95 \%}$ was also greater than 1 when the radius was within $[12,18]$ and mesh sizes were $\{16 \times 32,24 \times 24,24 \times 16,24 \times 32\}$, where $N \times M$ represents the spherical discretization grid with $N$ samples along the zenithal direction and $M$ samples along the azimuthal direction.

\section{Interobserver variability analysis}

The interobserver variability of the manually extracted $\mathrm{LV}$ volumetric indices was $15.0 \pm 17.2 \mathrm{~mL}$,
$13.4 \pm 13.5 \mathrm{~mL}, 12.4 \pm 11.3 \mathrm{~mL}$ and $7.0 \pm 5.5 \%$ for the EDV, ESV, SV and EF, respectively (estimated as E [abs(Refi-Refj)], $\mu \pm \sigma$ ). The inter-observer variability of the LV volumetric indices estimated with our semiautomatic approach, using the proposed segmentation energy, was $8.2 \pm 7.1 \mathrm{~mL}, 3.8 \pm 3.3 \mathrm{~mL}, 7.3 \pm 6.8$ $\mathrm{mL}$ and $3.6 \pm 4.3 \%$ for the EDV, ESV, SV and EF, respectively (estimated as $\mathrm{E}[\mathrm{abs}($ User1-User2)], $\mu \pm \sigma$ ). One should note that the proposed fully automatic framework does not take user input on none of its steps, and thus has no inter-observer variability.

\section{DISCUSSION}

\section{Proposed energy}

Classical region-based energies usually aim to provide an optimal partition of the image without any kind of prior knowledge regarding the appearance of the object and background regions. This is evident in eqn (4), where the segmentation energy is taken as the square difference between the local means inside and outside the contour. While this behavior might be desirable in a generic application, the proposed segmentation energy takes advantage of a very simple assumption which clearly improves the segmentation performance. This is reflected in the tighter limits of agreement of the Bland-Altman analysis for the proposed energy, as shown in Table 3. By simply taking into account the darker appearance of the blood pool explicitly, it is possible to avoid segmentation errors arising from sub-optimal initialization, as illustrated in Figure 5. In these situations, the right ventricle endocardium at septal wall

Table 4. Segmentation performance of the proposed fully automatic approach

\begin{tabular}{lcccc}
\hline & \multicolumn{4}{c}{ Automatic analysis (automatic/proposed energy) } \\
\cline { 2 - 5 } Initialization/segmentation & $\mathrm{EDV}(\mathrm{mL})$ & $\mathrm{ESV}(\mathrm{mL})$ & $\mathrm{SV}(\mathrm{mL})$ & $\mathrm{EF}(\%)$ \\
\hline Correlation coefficient $(\mathrm{R})$ & 0.971 & 0.972 & 0.938 & 0.907 \\
Bias $(\mu)$ & -1.43 & 2.02 & -3.45 & -0.95 \\
LOA $(\mu \pm 1.96 \sigma)$ & {$[-24.6,21.8]$} & {$[-16.6,20.7]$} & {$[-23.2,16.3]$} & {$[-10.7,8.82]$} \\
\hline
\end{tabular}

$\mathrm{EDV}=$ end-diastolic volume; $\mathrm{ESV}=$ end-systolic volume; $\mathrm{SV}=$ stroke volume; $\mathrm{EF}=$ ejection fraction; LOA = Bland-Altman's limits of agreement. $p<0.05$, paired $t$-test. 
a
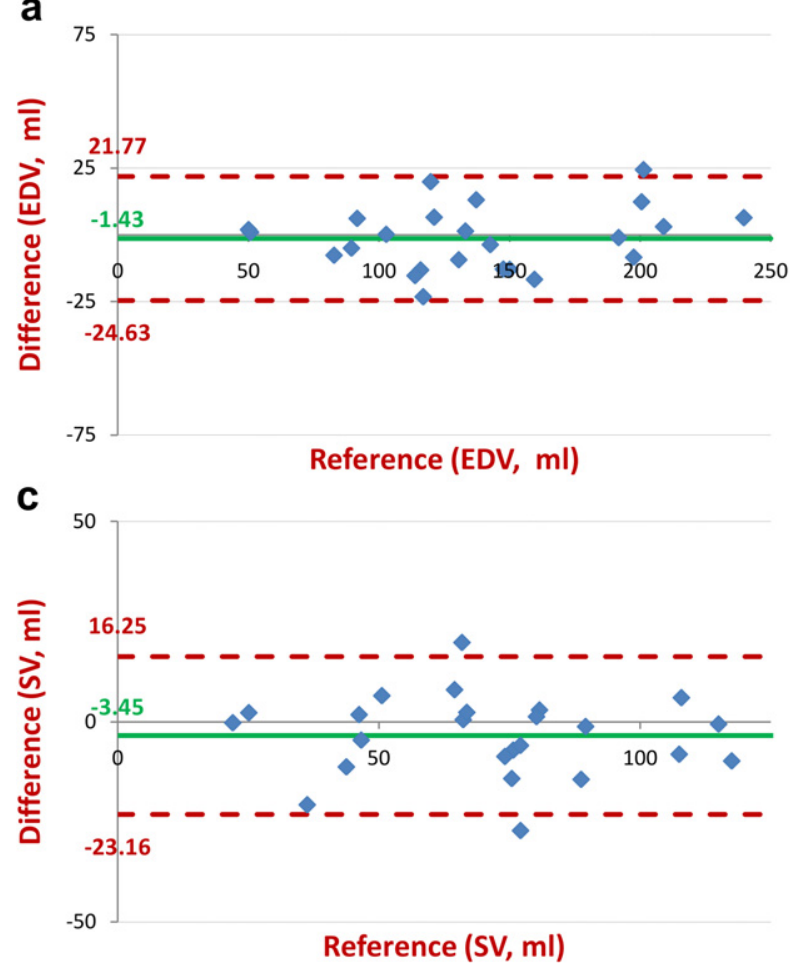

b

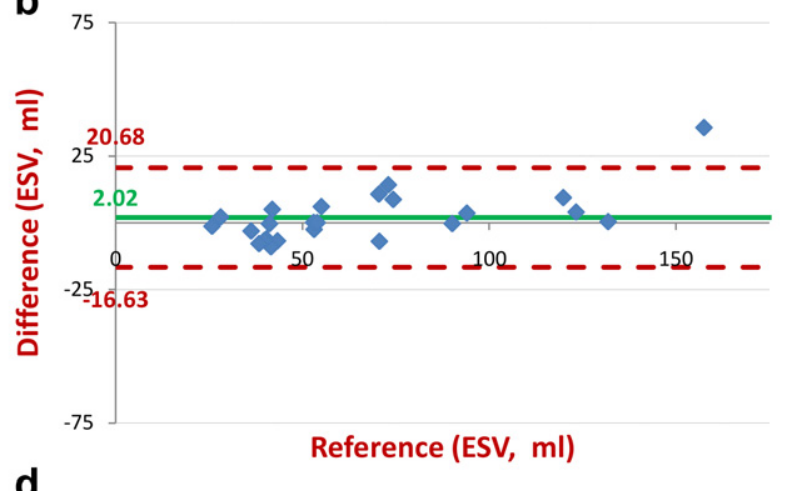

d

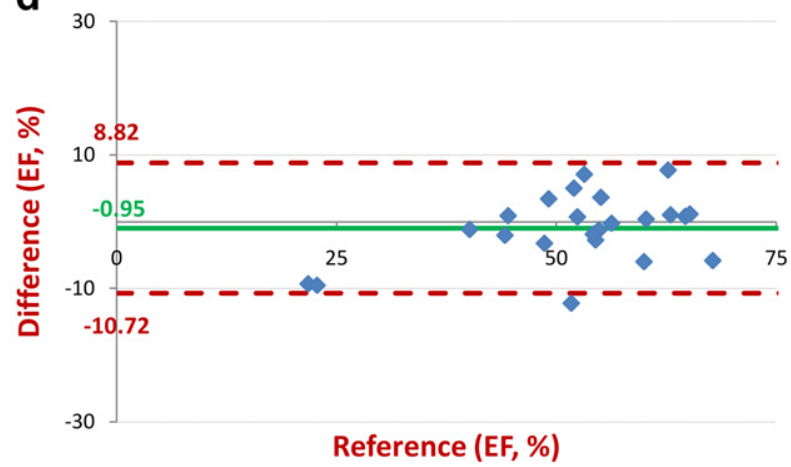

Fig. 6. Bland-Altman plots for EDV, ESV, SV and EF, comparing the measured errors against the reference values (green $=$ bias; red $=$ limits of agreement $(\mu \pm 1.96 \sigma)$.

appears as a strong candidate to the interface convergence in the classical local-based Yezzi energy, which leads to segmentation errors and reduced robustness.

\section{Automatic initialization algorithm}

Although the proposed segmentation energy offers promising results regarding left ventricular volumetric assessment, it still relies on manual initialization. However, comparing the results found in Table 3 and Table 4, it is clear that the proposed initialization scheme provides an appropriate ellipsoid to initialize the segmentation algorithm, since there is no reduction on the performance when compared with the semiautomated approach.

Bypassing manual initialization also avoids the introduction of user-dependent variability and allows taking full advantage of the important real-time image segmentation capability offered by BEAS. Indeed, the manual initialization time $(\sim 29 \mathrm{~s})$ was significantly reduced with the proposed initialization algorithm. The time of analysis required to extract all the desired cardiac indices fully automatically was around $1 \mathrm{~s}$, in a $\mathrm{C}++\mathrm{im}-$ plementation running in a standard laptop equipped with a $2.8 \mathrm{GHz}$ i7 Dual-Core processor. The details of the computation times referring to each step of the proposed algorithm are given in Table 5. Such an approach potentiates the design of online analysis tools that would provide real-time measurements of the LV volumes while the physician is scanning the patient and avoiding the cumbersome, offline manual analysis.

\section{Parameter sensitivity analysis}

Active contour-based segmentation frameworks usually imply a delicate balance of the diverse parameters involved in these algorithms. The stability of the proposed

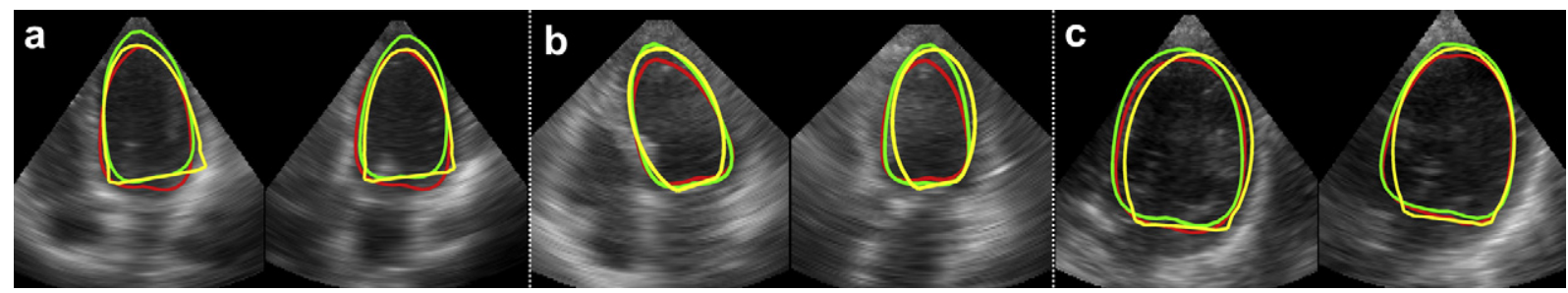

Fig. 7. Comparison between the proposed automatic initialization approach (yellow) and the manual initializations provided by two different users (red and green). 

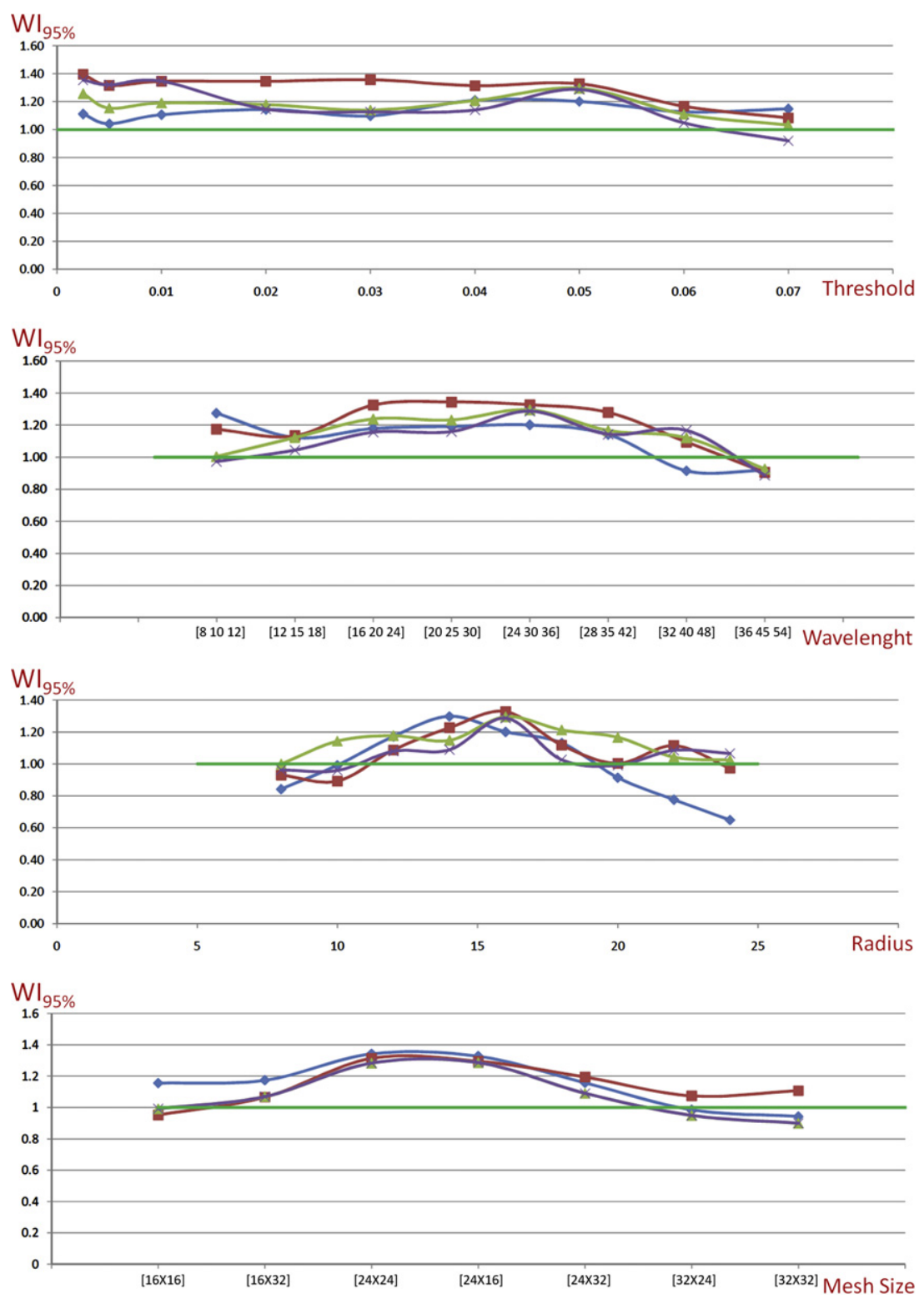

Fig. 8. Influence of the algorithm's parameters variation in the overall segmentation performance (top to bottom: edge detector threshold, phase-based filters' wavelength, local neighborhood size, spherical discretization grid size; blue = $\mathrm{EDV} ;$ red $=\mathrm{ESV}$; green $=\mathrm{SV}$; purple $=\mathrm{EF})$.

algorithm was tested by studying its robustness with respect to the variation of its parameters. The results show that accurate LV volume measurements are achievable over a wide range of values for the studied parameters. In fact, by imposing as performance quality condition that the $W I_{u p 95 \%}>1$ simultaneously for all the assessed volumetric indices, it is guaranteed that in this interval the agreement of the automatically extracted indices with the manual references is within the inter-observer range simultaneously for the EDV, ESV, SV and EF.

The results show that perturbations in the wavelengths used in the multi-scale phase-based edge detector do not have a major impact in the segmentation performance, although there is an optimal range. The threshold used in binarization of the edge detection map had little influence in the segmentation outcome. However, it was verified that higher values led to less edge information used in the LAX detection and ellipsoid fitting, ultimately reducing the performance of the initialization algorithm. The variation of the radius of the local neighborhood was found to have a stronger effect in the EDV estimation. This can be justified as a high value of $\rho$ leads to errors in the estimation of $v_{x}$, as it will account not only for myocardium but also for other tissue. This consequently affects 
Table 5. CPU computational cost of the different stages of the proposed framework

\begin{tabular}{lcccc}
\hline & ED initialization & ED segmentation & ES initialization & ES segmentation \\
\hline CPU processing time $(\mu \pm \sigma s)$ & $1.026 \pm 0.057$ & $0.059 \pm 0.002$ & $0.321 \pm 0.022$ & $0.028 \pm 0.001$ \\
\hline
\end{tabular}

$\mathrm{ED}=$ end-diastolic; $\mathrm{ES}=$ end-systolic.

the interface evolution toward the proper myocardial boundary. Note also that a too small $\rho$ value will limit the field of view of the interface during its evolution, affecting therefore the basin of convergence of the segmentation algorithm. The results of the variation of the discretization of $\Psi$ show that there is an intrinsic trade-off between a proper shape support and a smooth interface evolution. By having a spherical discretization grid too small, the segmented shapes are not able to properly capture all the shape details of the left ventricle. On the other hand, a too large spherical discretization grid will provide too much freedom in the interface evolution, since the support of each B-spline coefficient will be more local. This intrinsically reduces the robustness of the segmentation algorithm to noisy local means estimates.

It is also clear that the performance of the proposed method to extract both SV and EF is more unstable. Therefore, there might be a cumulative error effect between the ED and ES segmentation.

\section{Performance comparison}

Although the proposed approach offers promising results, a careful comparison with the values reported in the literature has been done. This comparison does not aim to be extensive, but rather informative to the reader on how the proposed fully automatic framework for LV volume analysis compares with existing relevant methods. An overview of the results of the proposed algorithm and its comparison with other methods reported in the literature is shown in Table 6. Nonetheless, it should be noted that a fair and quantitative comparison is not trivial, due to differences in patient population and image quality and due to different acquisition conditions and equipment. However, methods with a similar validation approach (i.e., where the segmentation results were compared with manual segmentation of RT3DE data) were selected.

\section{Study limitations}

The key limitation of the proposed framework for fully automatic LV volumetric assessment is the intrinsic assumption that the C-planes of the 3-D image volume correspond roughly to short-axis slices of the left ventricle. This is only true in apical acquisitions, and therefore the proposed pipeline will not automatically adapt to parasternal 3-D echocardiographic data, where the LAX is approximately perpendicular to the acquisition axis. Nonetheless, apical 3-D RT3DE data is far more commonly acquired in clinical practice.

It is also important to refer that the tuning of these parameters may be dataset dependent. Therefore, the scope of our work will be expanded toward data acquired with other ultrasound imaging systems, to test the robustness of our method with respect to the intrinsically different image characteristics provided by the ultrasound systems from the different medical vendors.

One should also note that the proposed deformation scheme is sub-optimal in its longitudinal component due to the effects caused by the mitral valve. In fact, if the ED segmentation result includes the mitral valve within the segmented surface, there may be an overestimation of the longitudinal deformation, since the proposed scheme favors an ES initialization where the mitral valve is left outside the segmented volume, to maximize the signed mean separation energy at this region. Nonetheless, efforts will be addressed toward the development of real-time tracking framework based on the existing segmentation algorithm, allowing thus to overcome this limitation.

Table 6. Segmentation performance comparison

\begin{tabular}{|c|c|c|c|c|c|c|c|}
\hline & \multirow[b]{2}{*}{ Exams \# } & \multicolumn{2}{|c|}{$\mathrm{EDV}(\mathrm{mL})$} & \multicolumn{2}{|c|}{ ESV (mL) } & \multicolumn{2}{|c|}{$\mathrm{EF}(\%)$} \\
\hline & & $\mathrm{BA}(\mu \pm 1.96 \sigma)$ & $\mathrm{R}$ & $\mathrm{BA}(\mu \pm 1.96 \sigma)$ & $\mathrm{R}$ & $\mathrm{BA}(\mu \pm 1.96 \sigma)$ & $\mathrm{R}$ \\
\hline Proposed method & 24 & $-1.4 \pm 23.2$ & 0.97 & $2.0 \pm 18.7$ & 0.97 & $-1.0 \pm 9.8$ & 0.91 \\
\hline Angelini et al. (2005) & 10 & $16.1 \pm 50.1$ & 0.63 & $-6.6 \pm 34.4$ & 0.63 & $-0.6 \pm 22.2$ & 0.45 \\
\hline Hansegard et al. (2007) & 21 & $-5.0 \pm 21$ & 0.91 & $6.2 \pm 19$ & 0.91 & $-7.7 \pm 12$ & 0.74 \\
\hline Muraru et al. (2010) & 23 & $-21.3 \pm 31.5$ & 0.89 & $-4.8 \pm 23$ & 0.83 & $-4.4 \pm 10.5$ & 0.75 \\
\hline Leung et al. (2010) & 99 & $1.47 \pm 35.4$ & 0.95 & $\mathrm{X}$ & $\mathrm{X}$ & $\mathrm{X}$ & $\mathrm{X}$ \\
\hline Leung et al. (2011) & 35 & $2.2 \pm 14.5$ & $\mathrm{X}$ & $1.4 \pm 11.8$ & $\mathrm{X}$ & $1.2 \pm 11.2$ & $\mathrm{X}$ \\
\hline Rajpoot et al. (2011) & 26 & $-5.1 \pm 48.5$ & $\mathrm{X}$ & $1.2 \pm 25.7$ & $\mathrm{X}$ & $0.7 \pm 14.1$ & $\mathrm{X}$ \\
\hline
\end{tabular}

$\mathrm{BA}=$ Bland-Altman analysis; $\mathrm{R}=$ Pearson product-moment correlation coefficient; \# = number of cases. 


\section{CONCLUSIONS}

In this article, a novel energy term is proposed to increase the robustness of the segmentation of RT3DE data, as well as a fast and accurate algorithm to initialize the segmentation method with a 3-D ellipsoid positioned near the endocardial interface.

The proposed framework provides an accurate and robust platform for fully automatic 3-D LV volumetric analysis. Furthermore, extraction of relevant volumetric cardiac indices takes around $1 \mathrm{~s}$. Therefore, the BEAS framework allows for a fast quantification of 3-D cardiac morphology and global function, facilitating its use in the clinical routine and in bed-side applications.

Acknowledgments-This work was partially funded by the region Rhône-Alpes, Tournesol French-Flemish cooperation programme and by the Portuguese Science Foundation (FCT PhD grant SFRH/BD/ 62230/2009).

\section{REFERENCES}

Angelini E, Homma S, Pearson G, Holmes J, Laine A. Segmentation of real-time three-dimensional ultrasound for quantification of ventricular function: A clinical study on right and left ventricles. Ultrasound Med Biol 2005;319:1143-1158.

Barbosa D, Bernard O, Friboulet D, Dietenbeck T, Houle H, D'hooge J. Fast 3-D echocardiographic segmentation using b-spline explicit active surfaces: A validation study in a clinical setting. Proc IEEE Int Ultrason Symp 2011;1854-1857. IUS2011.

Barbosa D, Dietenbeck T, Schaerer J, D'hooge J, Friboulet D, Bernard O. B-spline explicit active surfaces: An efficient framework for real-time 3D region-based segmentation. IEEE Trans Image Proc 2012a;211:241-251.

Barbosa D, Heyde B, Dietenbeck T, Houle H, Friboulet D, D'hooge J, Bernard O. Quantification of left ventricular volume and global function using a fast fully automated segmentation tool: Validation in a clinical setting. Int J Cardiovasc Imaging 2012b (in press), doi:10.1007/s10554-012-0103-8.

Barbosa D, Hristova K, Loeckx D, Rademakers F, Claus P, D'hooge J. 3D motion and strain estimation of the heart: Initial clinical findings. Proc SPIE Med Imaging 2010:762904-762904-9.

Bernard O, Friboulet D, Thévenaz P, Unser M. Variational B-Spline level-set: A linear filtering approach for fast deformable model evolution. IEEE Trans Image Proc 2009;18:1179-1191.

Bland J, Altman D. Statistical methods for assessing agreement between two methods of clinical measurement. Lancet 1986;327:307-310.

Bogaert J, Rademakers F. Regional nonuniformity of normal adult human left ventricle. Am J Physiol 2001;280:H610-H620.

Caiani EG, Corsi C, Zamorano J, Sugeng L, MacEneaney P, Weinert L, Battani R, Gutierrez JL, Koch R, de Isla LP, Mor-Avi V, Lang RM. Improved semiautomated quantification of left ventricular volumes and ejection fraction using 3-dimensional echocardiography with a full matrix-array transducer: Comparison with magnetic resonance imaging. J Am Soc Echocardiogr 2005;188:779-788.

Chalana V, Kim Y. A methodology for evaluation of boundary detection algorithms on medical images. IEEE Trans Med Imaging 1997;165: 642-652.

Hansegard J, Orderud F, Rabben S. Real-time active shape models for segmentation of 3D cardiac ultrasound. In: Kropatsch W, Kampel M, Hanbury A, (eds). Computer analysis of images and patterns. Vol. 4673 of lecture notes in computer science. Berlin/Heidelberg: Springer; 2007. p. 157-164.

Hansegard J, Urheim S, Lunde K, Malm S, Rabben S. Semiautomated quantification of left ventricular volumes and ejection fraction by real-time three-dimensional echocardiography. Cardiovasc Ultrasound 2009;71:18.
Hasegawa H, Kanai H. High-frame-rate echocardiography using diverging transmit beams and parallel receive beamforming. J Med Ultrason 2011;383:129-140.

Jacobs LD, Salgo IS, Goonewardena S, Weinert L, Coon P, Bardo D, Gerard O, Allain P, Zamorano JL, de Isla LP, Mor-Avi V, Lang RM. Rapid online quantification of left ventricular volume from real-time three-dimensional echocardiographic data. Eur Heart J 2006;274:460-468.

Jenkins C, Bricknell K, Hanekom L, Marwick TH. Reproducibility and accuracy of echocardiographic measurements of left ventricular parameters using real-time three-dimensional echocardiography. J Am Coll Cardiol 2004;444:878-886.

Kuznetsova T, Herbots L, Thijs L, Richart T, D'hooge J, Fagard R, Herregods M, Staessen J. Left ventricular strain and strain rate in a general population. Eur Heart J 2008;29:2014-2023.

Lankton S, Tannenbaum A. Localizing region-based active contours. IEEE Trans Image Proc 2008;1711:2029-2039.

Leung K, Bosch J. Automated border detection in three-dimensional echocardiography: Principles and promises. Eur J Echocardiogr 2010;112:97-108.

Leung K, Danilouchkine M, van Stralen M, de Jong N, van der Steen A, Bosch J. Left ventricular border tracking using cardiac motion models and optical flow. Ultrasound Med Biol 2011;374:605-616.

Leung K, van Stralen M, van Burken G, de Jong N, Bosch J. Automatic active appearance model segmentation of 3-D echocardiograms. Proc IEEE Int Symp Biomed Imaging 2010;320-323. ISBI2010.

Li B, Acton S. Automatic active model initialization via poisson inverse gradient. IEEE Trans Image Proc 2008;178:1406-1420.

Madore B, White P, Thomenius K, Clement G. Accelerated focused ultrasound imaging. IEEE Trans Ultrason Ferroelectr Freq Control 2009;5612:2612-2623.

Mor-Avi V, Jenkins C, Kühl HP, Nesser HJ, Marwick T, Franke A, Ebner C, Freed BH, Steringer-Mascherbauer R, Pollard H, Weinert L, Niel J, Sugeng L, Lang RM. Real-time 3-dimensional echocardiographic quantification of left ventricular volumes: Multicenter study for validation with magnetic resonance imaging and investigation of sources of error. JACC 2008;4:413-423.

Muraru D, Badano L, Piccoli G, Gianfagna P, Del Mestre L, Ermacora D, Proclemer A. Validation of a novel automated border-detection algorithm for rapid and accurate quantitation of left ventricular volumes based on three-dimensional echocardiography. Eur J Echocardiogr 2010;114:359-368.

Rajpoot K, Grau V, Noble J. Local-phase based 3-D boundary detection using monogenic signal and its application to real-time 3-D echocardiography images. Proc IEEE Int Symp Biomed Imaging 2009;783-786. ISBI2010.

Rajpoot K, Grau V, Noble JA, Becher H, Szmigielski C. The evaluation of single-view and multi-view fusion 3-D echocardiography using image-driven segmentation and tracking. Med Image Analysis 2011;154:514-528.

Saito K, Okura H, Watanabe N, Hayashida A, Obase K, Imai K, Maehama T, Kawamoto T, Neishi Y, Yoshida K. Comprehensive evaluation of left ventricular strain using speckle tracking echocardiography in normal adults: Comparison of three-dimensional and two-dimensional approaches. J Am Soc Echocardiogr 2009;229: $1025-1030$.

Soliman OI, Kirschbaum SW, van Dalen BM, van der Zwaan HB, Delavary BM, Vletter WB, van Geuns RJM, Cate FJT, Geleijnse ML. Accuracy and reproducibility of quantitation of left ventricular function by real-time three-dimensional echocardiography versus cardiac magnetic resonance. Am J Cardiol 2008; 1026:778-783.

Soliman OI, Krenning BJ, Geleijnse ML, Nemes A, van Geuns RJ, Baks T, Anwar AM, Galema TW, Vletter WB, Cate FJT. A comparison between qlab and tomtec full volume reconstruction for real time three-dimensional echocardiographic quantification of left ventricular volumes. Echocardiography 2007;249:967-974.

Tauber C, Batatia H, Ayache A. Quasi-automatic initialization for parametric active contours. Pattern Recogn Lett 2010;31:83-90.

Tong L, Gao H, Choi HF, Dhooge J. Multi-transmit beam forming for fast cardiac imaging. Proc IEEE Int Ultrason Symp 2011; 140-143. IUS2011. 
Unser M. Splines: A perfect fit for signal and image processing. IEEE Signal Proc Magazine 1999;166:22-38.

Uzumcu M, van der Geest R, Swingen C, Reiber J, Lelieveldt B. Time continuous tracking and segmentation of cardiovascular magnetic resonance images using multidimensional dynamic programming. Invest Radiol 2006;411:52-62.

van Stralen M, Leung K, Voormolen M, de Jong N, van der Steen A, Reiber J, Bosch J. Time continuous detection of the left ventricular long axis and the mitral valve plane in 3-d echocardiography. Ultrasound Med Biol 2008;34:196-207.

Yezzi A, Tsai A, Willsky A. A fully global approach to image segmentation via coupled curve evolution equations. J Visual Commun Image Represent 2002;13:195-216.

\section{APPENDIX: SEGMENTATION ENERGY DERIVATION}

The classical mean separation energy has been proposed by Yezzi et al. (2002) to segment an object from the image background by maximizing the intensity means between the different regions inside and outside an evolving contour. By considering $I(\mathbf{x})$ a $N$-dimensional image partitioned into two different regions $\Omega_{i n}$ and $\Omega_{\text {out }}$, the corresponding criterion can be written as:

$$
E_{Y}=-\frac{1}{2}(u-v)^{2}
$$

where $u$ and $v$ represent the mean inside and outside the evolving interface respectively.

In the present work, the fact that the inside region (blood pool) should be darker than the outside one (tissue area) is explicitly taken as a prior information. Thus, we propose to minimize the following energy functional:

$$
E_{G}=u-v,
$$

Moreover, as the statistics are not consistent over the endocardial border, we propose to localize this criterion. The underlying energy model states as:

$$
E_{L}=\int_{\Omega} \delta_{\phi}(\mathbf{x}) \int_{\Omega} B(\mathbf{x}, \mathbf{y}) F_{L}\left(\mathbf{y}, H_{\phi}(\mathbf{y})\right) \mathrm{d} \mathbf{y} \mathrm{d} \mathbf{x},
$$

and

$$
F_{L}\left(\mathbf{y}, H_{\phi}(\mathbf{y})\right)=\left(u_{x}-v_{x}\right),
$$

where $u_{x}$ and $v_{x}$ are the local means inside and outside the interface at the position $\mathbf{x}$ and $\phi(\mathbf{x})=\psi\left(\mathbf{x}^{*}\right)-x_{1}$. Now getting use of the BEAS framework proposed in the work of Barbosa et al. (2012a), the proposed energy gradient with respect to a given B-spline coefficient $c\left[k_{i}\right]$ can be directly expressed by:

$$
\frac{\partial E_{L}}{\partial c\left[\mathbf{k}_{\mathbf{i}}\right]}=\int_{\Gamma} \bar{g}_{L}\left(\mathbf{x}^{*}\right) \beta^{d}\left(\frac{\mathbf{x}^{*}}{h}-\mathbf{k}_{i}\right) \mathrm{d} \mathbf{x}^{*},
$$

where

$$
\bar{g}_{L}\left(\mathbf{x}^{*}\right) \delta_{\phi}\left(\mathbf{x}^{*}\right)=\frac{\partial \bar{F}_{L}\left(\mathbf{x}^{*}, H_{\phi}\left(\mathbf{x}^{*}\right)\right)}{\partial \phi} .
$$

Using standard calculus of variation, we obtain:

$$
\bar{g}_{L}\left(\mathbf{x}^{*}\right)=\left(\frac{\left(\bar{I}\left(\mathbf{x}^{*}\right)-u_{x}\right)}{A_{u}}+\frac{\left(\bar{I}\left(\mathbf{x}^{*}\right)-v_{x}\right)}{A_{v}}\right) .
$$

Now getting use of (A.6) and (A.7), we directly obtain eqn (7). 